\title{
Human herpesvirus-6-associated acute lymphadenitis in immunocompetent adults
}

\author{
Irina Maric ${ }^{1}$, Ron Bryant ${ }^{2}$, Mones Abu-Asab ${ }^{1}$, Jeffrey I Cohen ${ }^{3}$, Angelica Vivero ${ }^{1}$, \\ Elaine S Jaffe ${ }^{1}$, Mark Raffeld ${ }^{1}$, Maria Tsokos ${ }^{1}$, Peter M Banks ${ }^{2}$ and Stefania Pittaluga ${ }^{1}$ \\ ${ }^{1}$ Laboratory of Pathology, National Cancer Institute, National Institutes of Health; Bethesda, MD, USA; \\ ${ }^{2}$ Department of Pathology, Carolinas Medical Center, Charlotte, NC, USA and ${ }^{3}$ National Institute of Allergy \\ and Infectious Diseases, National Institutes of Health, Bethesda, MD, USA
}

\begin{abstract}
In contrast to other causes of herpetic lymphadenitis, the histological features associated with human herpesvirus-6 (HHV-6) infection have remained elusive since its discovery in 1986. We describe the histologic and phenotypic changes associated with acute HHV-6 lymphadenitis in two immunocompetent adults who presented with fever, fatigue, generalized lymphadenopathy, and elevated liver enzymes. Serologic tests for human immunodeficiency virus, acute Epstein-Barr virus, and cytomegalovirus infection were negative. Lymph node biopsies were consistent with viral lymphadenitis. Intranuclear and cytoplasmic inclusions were identified in CD4-positive T lymphocytes in expanded paracortical areas. Immunohistochemical staining with monoclonal antibody to the HHV-6 gp60/110 kDa envelope glycoprotein showed that the inclusions were positive for viral antigen. Electron microscopy demonstrated numerous viral particles in the cytoplasm and nucleus, characteristic of Herpesviridae family. Clustering of viral particles was observed, which has previously been reported only in infected tissue culture cells. PCR followed by sequencing of DNA extracted from the lymph nodes identified the virus as HHV-6, type B. This is the first report that documents distinctive histologic features of HHV-6 lymphadenitis and demonstrates that the cells harboring the virus in vivo are CD4-positive T lymphocytes.
\end{abstract}

Modern Pathology (2004) 17, 1427-1433. doi:10.1038/modpathol.3800179

Keywords: CD4-positive cells; electron microscopy; HHV-6; lymphadenitis; T lymphocytes

Human herpesvirus-6 (HHV-6) is ubiquitous in the human population throughout the world, with seroconversion occurring early in life. Primary infection with HHV-6 in young children may cause exanthem subitum ${ }^{1}$ and acute febrile illness. In adults, it has been associated with hepatitis, ${ }^{2}$ encephalitis, ${ }^{3}$ and a mononucleosis-like syndrome. ${ }^{4}$ Two variants of HHV-6 exist (A and B), which have colinear and highly homologous genomes. ${ }^{5}$ Like all other human herpesviruses, HHV-6 remains latent in host cells after primary infection. Reactivation usually occurs only in immunocompromised states, and recently HHV-6 has been recognized as an opportunistic pathogen in patients with acquired immune deficiency syndrome and in transplant recipients. ${ }^{6}$ Several studies have also suggested association between HHV-6 infection and certain

Correspondence: Dr S Pittaluga, Hematopathology Section, Laboratory of Pathology, National Cancer Institute, National Institutes of Health, Building 10/ Room 2N202, 10 Center Drive, Bethesda, MD, 20892, USA.

E-mail: stefpitt@mail.nih.gov

Received 9 January 2004; revised and accepted 23 April 2004 lymphoproliferative disorders. DiLuca et $a l^{7}$ reported the presence of HHV-6, variant B, in about one-third of Hodgkin's lymphomas. Luppi et $a l^{8}$ detected HHV-6 sequences in lymph node biopsies of seven out of 12 cases of angioimmunoblastic lymphadenopathy with dysproteinemia. HHV-6 was detected in tissues involved by sinus histiocytosis with massive lymphadenopathy. ${ }^{9}$ While these findings are intriguing, it is not certain that HHV-6 has a causal role in malignancy. While viral DNA has been detected in these tissues by PCR or DNA hybridization, the number of positive cells by immunohistochemistry has been usually less than $1 \%$, and no distinctive features were associated with HHV-6 infection in tissue sections.

It is still largely unknown which cell types harbor HHV-6 in lymphoid tissues in vivo. Immunohistochemical studies ${ }^{10}$ showed that HHV-6-positive cells in cases of reactive lymphadenopathy are plasma cells, histiocytes, and rare granulocytes. In vitro studies have shown that HHV-6 replicates and induces cytopathic effects in $\mathrm{CD}^{+}$-positive $\mathrm{T}$ lymphocytes, but it has been also shown to be tropic for other cells including CD8 ${ }^{+} \mathrm{T}$ cells, $\gamma \delta \mathrm{T}$ 
cells, B cells, natural killer cells, monocytes/macrophages, and megakaryocytes. ${ }^{11-15}$

In the present report, we describe two cases of immunocompetent adults who presented with fever and generalized lymphadenopathy, and on biopsy were found to have viral lymphadenitis with massive numbers of viral inclusions in CD4-positive $\mathrm{T}$ lymphocytes. The presence of the virus in these cells was confirmed by immunohistochemistry, and electron microscopy and molecular studies identified distinctive features of HHV-6B.

\section{Material and methods}

\section{Case Reports}

Case no. 1

A 28-year-old male student from Ghana, with no significant past medical history except for a remote history of malaria, was admitted to the hospital for evaluation of an acute febrile illness that began approximately 1 week before admission. Symptoms included high fever, headaches, myalgias, arthralgias, upper respiratory tract infection, and generalized lymphadenopathy. On admission, blood and throat cultures were obtained that were subsequently negative and he was started on ampicillin-sulbactam. The white blood cell (WBC) count was $6400 \mu \mathrm{l}^{-1}$ (30\% bands), hematocrit was 0.41 , and platelets $125000 \mu \mathrm{l}^{-1}$. The peripheral blood smear showed no atypical lymphocytes. The serum creatinine was $1.7(0.5-1.3 \mathrm{mg} / \mathrm{dl})$, BUN $8(6-21 \mathrm{mg} / \mathrm{dl})$, albumin 2.9 (3.5-4.9g/dl), CPK 3214 (52-380 U/l), AST 78 (5-35 U/l), ALT 147 (7-56 U/l), alkaline phosphatase 100 (38-126 U/l), and total bilirubin 0.3 $(0.2-1.3 \mathrm{mg} / \mathrm{dl})$. A malaria smear was negative. Serologies were negative for human immunodeficiency virus (HIV), hepatitis B and hepatitis C, and cytomegalovirus (CMV). Tests for cryptococcus, parvovirus, toxoplasmosis, and acid-fast bacilli were negative. The RPR was nonreactive. Epstein-Barr virus (EBV) serology showed positive titers for EBNA and VCA IgG; the VCA IgM was negative. FANA was negative, and complements were within normal limits.

Abdominal ultrasound showed splenomegaly. After ampicillin-sulbactam was begun, the patient developed a sandpaper-like generalized rash and peripheral blood eosinophilia. Antibiotic was stopped and a cervical lymph node biopsy was performed. The patient was subsequently discharged on Zitromax.

\section{Case no. 2}

An acutely ill 56-year-old African-American female was admitted with fever, rash, nausea, vomiting, diarrhea, and right upper quadrant abdominal pain. Her medical history was notable for a diagnosis of rheumatoid arthritis approximately 4 weeks earlier and initiation of the medications Sulfasalazine, Valdecoxib, and Diclofenac. Physical examination was remarkable for a maculopapular rash on the right side of her face and right upper quadrant tenderness. An admission diagnosis of hepatitis was based on a bilirubin of $4.3(0.2-1.0 \mathrm{mg} / \mathrm{dl})$, alkaline phosphatase of 191 (39-117 U/l), AST of 76 (1-40 U/l), and ALT of 149 (9-49 U/l.) Initial WBC was $8200 \mu \mathrm{l}$ with $47 \%$ neutrophils and $35 \%$ bands.

Patient's rash, which started 2 days prior to admission, progressed over the course of her hospitalization to be exfoliative and involved her entire body. She developed axillary and supraclavicular lymphadenopathy and palpable hepatosplenomegaly. CT scan of the abdomen showed porta-hepatis lymphadenopathy and suggested cholecystitis. After five relatively stable days her WBC counts increased to $40000 \mu \mathrm{l}$ with a marked atypical lymphocytosis accounting for the elevation. Eosinophilia was not present. AST increased to 1642 (10-40 U/l), ALT to 797 (9-49 U/l), and bilirubin to $12(0.2-1.0 \mathrm{mg} / \mathrm{dl}$.) Serologies were negative for HIV, hepatitis A, hepatitis B, hepatitis C, CMV, and HTLV I \& II. EBV virus serologies were positive for VCA and EBNA IgG. EBV EA IgG and VCA IgM were negative. Varicella/zoster IgG was positive and IgM was negative. Blood and respiratory cultures were negative.

The patient developed worsening liver failure with confusion, renal failure, disseminated intravascular coagulation, and pleural effusion. An exploratory laparotomy with liver and porta-hepatis lymph node biopsies were carried out hospital on day 12. Bone marrow biopsy was also performed. Based on the recognition of viral inclusions in the biopsies acyclovir was started, but patient expired with worsening liver failure and encephalopathy 17 days after admission. Permission for autopsy was not obtained.

\section{Histological Analysis}

The tissue biopsies were fixed in 10\% phosphatebuffered formalin solution and paraffin-embedded according to conventional procedures. Sections ( $4 \mu \mathrm{m}$ thick) were cut and stained with hematoxylin-eosin (H\&E) using standard procedures.

\section{Immunohistochemical Analysis}

Immunohistochemical studies were performed on formalin-fixed, paraffin-embedded sections using standard immunoperoxidase staining procedures with an automated immunostainer (Ventana Medical System, Tucson, AZ, USA), according to the manufacturer's instruction as previously described. ${ }^{16}$ The antibody panel for lymph node staining in Case no. 1 included CD20, CD3, CD8, Alk-1, HSV-1, CMV (clone CCH2) (Dako, Carpinteria, CA, USA), CD4, CD56, CD25 (Novocastra Laboratories, Newcastle, UK), HHV-8 (ORF-73)(Advanced Biotechnologies Inc., Columbia, MD, USA), and HHV-6 gp60/110 
envelope glycoprotein, which recognizes type A and B (Biodesign Inter, Saco ME, USA). HHV-6 monoclonal antibody was applied for $1 \mathrm{~h}$ at RT (dilution 1:50), after antigen retrieval in a microwaveable pressure cooker (Nordic Ware) containing $1.5 \mathrm{l}$ of $10 \mathrm{mM}$ citrate buffer ( $\mathrm{pH}$ of 6.0 ) with $0.1 \%$ Tween 20, and microwaved (Model R4A80, Sharp Electronics, Rahwah, NJ, USA) for $8 \mathrm{~min}$ at $700 \mathrm{~W}$. It was visualized using EnvisionPlus (Dako, Carpinteria, CA, USA) according to the manufacturer's instructions.

The antibody panel for lymph node staining in Case no 2 included CD20, CD3, CD30, CD34, CD43, CD45RO, CD68, EBV, HSV-I \& II, and CMV. Additional immunohistochemical stains were performed for Varicella-zoster (Phenopath Laboratories, Seattle, WA, USA).

\section{Electron Microscopic Analysis}

Lymph node tissue was removed from paraffin blocks, deparaffinized overnight in xylene, rehydrated in PBS, and postfixed in osmium tetroxide $(0.5 \%)$,. dehydrated, and embedded into Maraglas epoxy resin. Ultrathin sections (90 nm) were made and double-stained with uranyl acetate and lead citrate, and viewed with a Philips CM10 transmission electron microscope.

\section{Molecular Analysis}

In Case no 1, genomic DNA was extracted from a formalin-fixed, paraffin-embedded cervical lymph node tissue block. PCR was amplified for detection of T-cell receptor gamma chain gene rearrangements. Two separate reactions were performed, one with primers Vg101, Vg11, and Jg12 and a second with primers Vg 101, Vg11, and Jp12, according to published methods. ${ }^{17}$ The products were analyzed by acrylamide gel electrophoresis. PCR for HTLV-I and II was performed with two sets of primers: one directed to sequences in the TAX gene and the other to sequences in the POL gene. The products were transferred to NYTRAN and hybridized with probes directed to internal sequences of the TAX and POL I and POL II genes. PCR for HHV-6 and HHV-7 was performed with $500 \mathrm{ng}$ of DNA extracted from the lymph node using specific primers. Primer sequences for HHV-6 were 5'-CTGTATTAGTTGTGCG TCTC- $3^{\prime}$ and $5^{\prime}$-CATCGACATCGGGATGCTTC- $3^{\prime}$ and for HHV-7 were $5^{\prime}$-TATCCCAGCTGTTTTCATA TAGTAAC- $3^{\prime}$ and $5^{\prime}$-GCCTTGCGGTAGCACTAGAT TTTTTG- ${ }^{\prime}$. The PCR conditions used were one cycle at $94^{\circ} \mathrm{C}$ for $5 \mathrm{~min}$; 35 cycles of $94^{\circ} \mathrm{C} \times 1 \mathrm{~min}$, $55^{\circ} \mathrm{C} \times 2 \mathrm{~min}, 72^{\circ} \mathrm{C} \times 3 \mathrm{~min}$; followed by one cycle of $72^{\circ} \mathrm{C} \times 7 \mathrm{~min}$. Negative controls included $500 \mathrm{ng}$ of DNA from peripheral blood mononuclear cells from 4 patients with unrelated conditions. A $1.7 \mathrm{~kb}$ band was present only with the HHV-6 primers using
DNA from the lymph node biopsy. The band was excised from the gel and DNA was sequenced.

In Case no. 2, formalin-fixed, paraffin-embedded sections of the lymph node were assayed by Colorimetric Microtiter Plate PCR for HHV-6, HHV-7, HHV-8, and EBV as previously described..$^{18}$

\section{In Situ Hybridization Studies}

In situ hybridization studies for EBV were performed using INFORM EBER probe and ISHiView Blue detection system on an automated system (BenchMark, Ventana Systems, Tucson, AZ, USA) in Case no. 1.

\section{Results}

Histological analysis of lymph nodes obtained from both patients revealed marked paracortical expansion with a polymorphous population of atypical cells of varying sizes. Some cells contained large centrally placed nucleoli and resembled immunoblasts. Many of the atypical cells, particularly in the subcapsular areas, contained large, eosinophilic, nuclear, and/or cytoplasmic inclusions, suggestive of a viral etiology (Figure 1a, Case no. 1). Scattered eosinophils and plasma cells were present and residual small follicles were seen.

Immunohistochemical analysis with antibody against HHV-6 envelope glycoprotein (gp60/ $110 \mathrm{kDa}$ ) highlighted numerous viral inclusions in the paracortical areas of lymph nodes (Figure 1b). The inclusions were mostly cytoplasmic with a Golgi distribution. The majority of cells in the paracortex, including the atypical cells containing the inclusions, were CD3-positive (Figure 1c) and CD4-positive (Figure 1d) T lymphocytes. CD20 highlighted only residual follicles and rare scattered larger cells in paracortical areas, consistent with immunoblasts. Antibody to CD8 stained only a minority of small, scattered lymphocytes. Staining with CD25 antibody was negative in atypical cells and highlighted small, activated T cells. ALK-1 and CD56 immunostains were negative. Immunohistochemistry was also negative for HSV-1, CMV, or HHV-8.

In situ hybridization study for EBV using an EBER-1 probe was performed on Case no. 1 and was negative.

Histological analysis of liver biopsy from Case no. 2 showed an intense and expansile portal inflammatory reaction with viral inclusions in lymphoid cells and hepatocytes (Figure 1f). There was extensive periportal hepatocyte necrosis and loss. A bone marrow exam showed vaguely granulomatous lymphohistiocytic aggregates, a large population of reactive lymphoid cells, and maturing hematopoiesis. Lymphoid cells in association with lymphohistiocytic aggregates showed rare viral inclusions. 

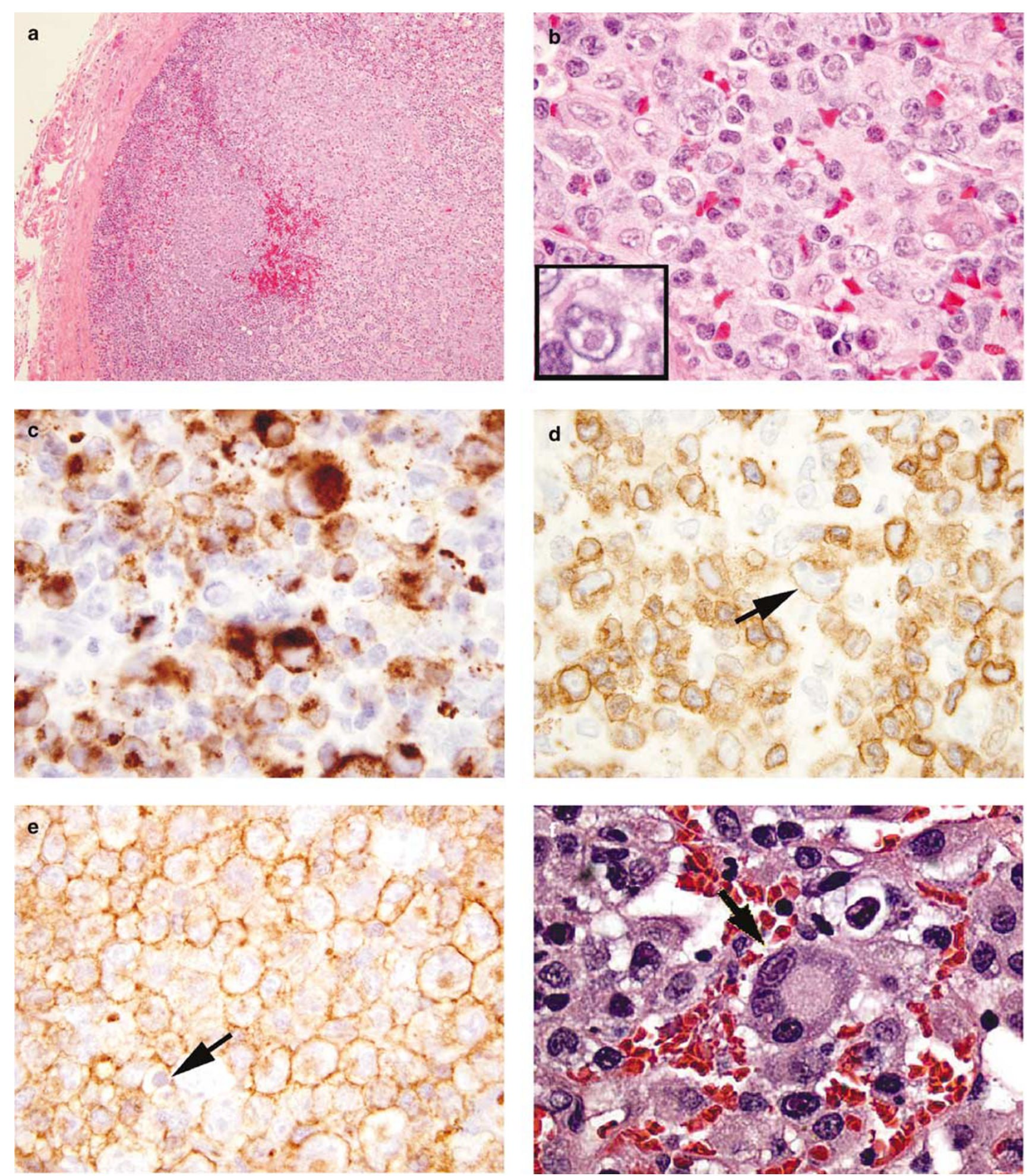

Figure 1 Histological and immunohistochemical findings. (a) and (b) HE-stained sections of the cervical lymph node from the Case no. 1 with markedly expanded paracortical areas containing a polymorphous population of atypical cells of varying sizes. Many of the cells contain large, eosinophilic, nuclear, and/or cytoplasmic inclusions, suggesting viral etiology. (c) Immunostaining for HHV-6 revels numerous positive atypical cells. Cytoplasmic inclusions are positive; however, antibody does not stain nuclear inclusions. (d) and (e). Immunohistochemical stains reveal that atypical cells, including cells with inclusions (arrows), are positive for CD3 (d) and CD4 (e) and negative for CD8 (not shown). (f) H\&E-stained section of the liver biopsy from the Case No 2 demonstrating viral inclusions. 
Transmission electron microscopy (TEM) was performed in both cases and revealed numerous viral particles consistent with HHV-6 (Figures 2a and b, Case no. 1) in a subset of lymphocytes. Particles were present both in the nucleus and in the cytoplasm of infected cells. In the nucleus, empty capsids or nucleocapsids lacking a tegument and an envelope were seen (solid arrows). In the cytoplasm, nucleocapsids were surrounded by a thick tegument (arrowheads) and many of them had an envelope with spikes (open arrows and inset) (Figure 2a). Intranuclear virions were of smaller size $(108 \mathrm{~nm})$ than cytoplasmic virions (average size $139 \mathrm{~nm}$ excluding spikes). The cytoplasmic virions occurred singly or in clusters. Clustered virions were sometimes seen in membrane-bound vacuoles (Figure $2 b)$.

PCR of DNA extracted from the lymph nodes of both patients, using primers specific for HHV-6 and HHV-7 showed a $1.7 \mathrm{~kb}$ band only with HHV-6 primers. The band was excised from the gel in Case no. 1 and sequence analysis showed that it corresponded to HHV-6, type B. In addition, in Case no. 1 molecular studies for T-cell receptor gene rearrangements were negative and PCR analysis failed to detect HTLV-I and HTLV-II.

\section{Discussion}

In this report, we describe the unique features of HHV-6-associated lymphadenitis in two adults presenting with an acute febrile illness. Lymph node biopsies showed marked paracortical expansion due to a proliferation of large atypical cells resembling immunoblasts. Numerous intranuclear and cytoplasmic inclusions were seen in expanded paracortical areas. Immunohistochemical studies showed that the cells containing the inclusions were CD3/CD4-positive T lymphocytes, and electron microscopic analysis demonstrated numerous viral particles in the cytoplasm and nuclei of the infected cells, consistent with the herpesvirus family. Immunohistochemical staining for HHV-6 using an antibody against the envelope glycoprotein gp60/110 kDa was positive, while immunohistochemical stains for HSV-1, CMV, EBV, and HHV-8 were negative. PCR and sequence analysis confirmed HHV-6 with a type B genotype.

Figure 2 Transmission electron microscopy (TEM) micrographs of infected cervical lymph node. (a) Naked nucleocapsids are present in the nucleus (solid arrows). Nuleocapsids surrounded by a thick fuzzy coat (tegument) are present in the cytoplasm (arrowheads). Some of the tegumented nucleocapsids in the cytoplasm are also surrounded by an outer envelope with spikes (open arrows). Mature virions composed of the nucleocapsid, the tegument and the envelope with spikes are shown in the inset. Bar equals $1 \mu \mathrm{m}$. (b) Several clusters of mature virions in membrane-bound vacuoles (endosomes) are present in the cytoplasm of this cell. Bar equals $0.5 \mu \mathrm{m}$.
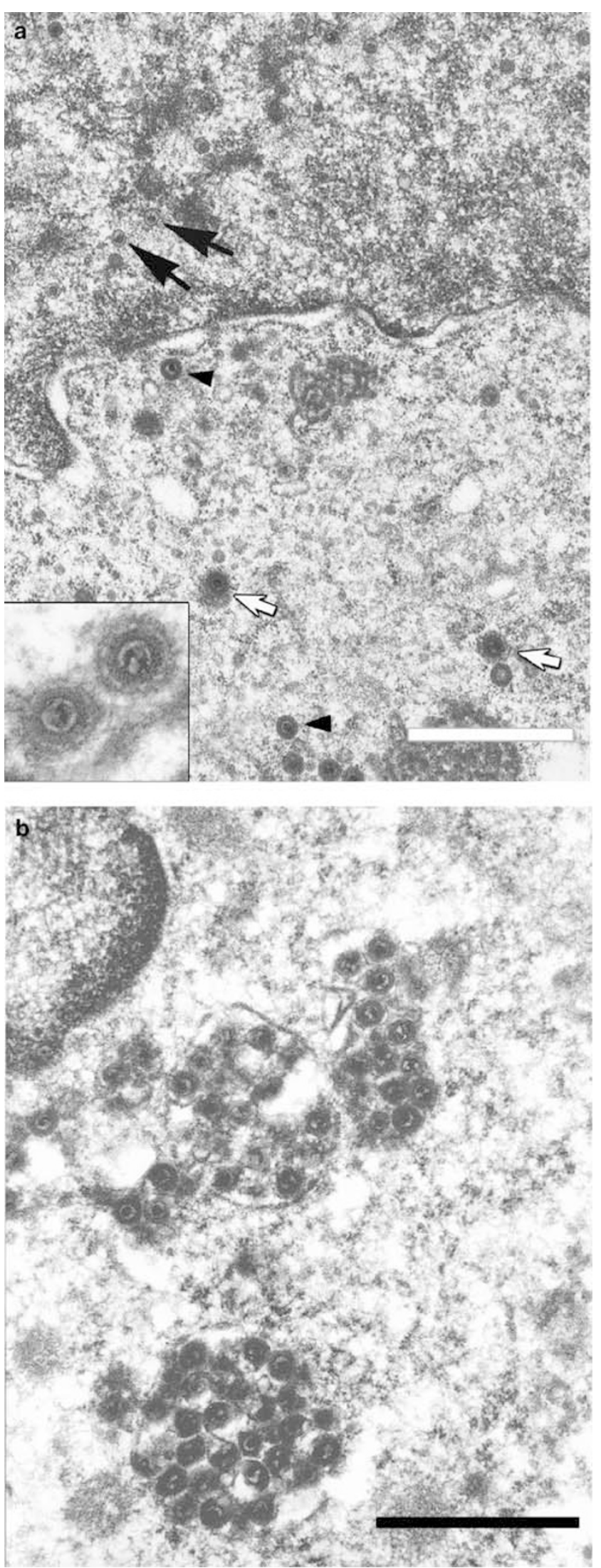
The florid and monomorphic expansion of the paracortex has not been described in the previous report of HHV6-associated lymphoproliferative disorders. ${ }^{10}$ Initially, the atypical cytological features of the virally infected cells raised the possibility of involvement by non-Hodgkin's lymphoma with immunoblastic features. However, the lack of an aberrant phenotype and absence of evidence of a clonal T-cell process did not support this diagnosis. We also considered CMV lymphadenitis in the differential diagnosis. CMV infection is usually associated with viral inclusions in endothelial cells. However, in rare instances CMV infection of T cells has been described. ${ }^{19}$ Such cells may contain characteristic owl's eye inclusion bodies in expanded paracortical areas.

Since most people become infected with HHV-6 during childhood, reports of symptomatic HHV-6 infections in adults are relatively uncommon. Mononucleosis-like illness due to HHV-6 with fatigue, cervical lymphadenopathy, sore throat, and elevated liver enzymes lasting several weeks has been reported. ${ }^{4}$ HHV-6 type B was responsible for illness in most of the prior reports in immunocompetent patients, as well as in our cases, while HHV-6 type A is usually detected in patients with HIV infection. Primary infection with HHV-6 is almost ubiquitous in infancy, and is associated with febrile illness, including exanthem subitum (also known as roseola infantum or sixth disease). ${ }^{1} \mathrm{HHV}-6$ remains in the host for life after primary infection. While the actual site of latency has yet to be established, monocytes, ${ }^{14}$ macrophages, and early bone marrow progenitor cells ${ }^{20}$ are likely sites for persistence.

In this study, we demonstrated the presence of HHV-6 viral particles in CD4-positive T lymphocytes in lymph node biopsies from symptomatic patients. This is the first report to demonstrate massive infection of CD4-positive lymphocytes in symptomatic, apparently immunocompetent adults. CD8-positive cells were not infected, although there are reports that CD8-positive $\mathrm{T}$ lymphocytes can also be infected with HHV-6 in vitro. ${ }^{21}$

EM features of the viral particles were characteristic for HHV-6. So far, ultrastructural studies of the HHV-6 virus in human biopsy material have been very limited. ${ }^{22,23}$ The most characteristic feature that distinguishes HHV-6 and HHV-7 from other herpesviruses is a prominent tegument layer in viral particles present in the cytoplasm, ${ }^{24}$ as shown in the present case. Detailed ultrastructural analysis of an HHV-6-infected human T-lymphoid cell line ${ }^{25}$ showed that HHV-6, like other herpesviruses, acquires an envelope at the inner nuclear membrane and loses its initial envelope at the outer nuclear membrane (de-envelopment). Naked nucleocapsids are then released into the cytoplasm and acquire a tegument and a new envelope with spike glycoproteins (re-envelopment). Our finding of both unenveloped and enveloped tegumented nucleocapsids in the cytoplasm is consistent with the reported de- envelopment/re-envelopment maturation pathway of the virus. In addition, we show for the first time in human tissue clustering of mature HHV-6 virions in membrane-bound vacuoles, thought to represent endosomes. Virus-containing vacuoles have been previously reported at the peak of virus production in the cytoplasm of tissue culture cells infected with pseudorabies virus ${ }^{26}$ and endosomal inclusions of virions were observed in tissue culture cells infected with HHV-6 ${ }^{25}$ and pseudorabies virus. ${ }^{26}$ Endosomes have been considered to facilitate transport of the virus to the plasma membrane ${ }^{26}$ or to serve as sites of viral degradation. ${ }^{25}$ While the function of the clustered virions in the membrane-bound vacuoles is unknown, their abundance in present cases supports the diagnosis of an active HHV-6 infection.

An interesting association in Case No 2 is the diagnosis of rheumatoid arthritis 4 weeks prior to patient's admission and medication with several drugs, including sulfasalazine. Sulfasalazine is one of several drugs associated with drug-induced hypersensitivity syndrome, which has been hypothesized to cause activation of $\mathrm{T}$ lymphocytes followed by reactivation and efficient replication of HHV-6. ${ }^{27}$ Thus, drug hypersensitivity might precipitate HHV-6-associated viral lymphadenitis in individuals with past, rather than primary, exposure to HHV-6.

\section{Note}

During the revision of the manuscript, we encountered a third case of HHV-6-associated acute lymphadenitis in an immunocompetent adult. The patient was a 34-year-old woman who developed erythematous induration on both feet after insect bite. Patient was placed on multiple antibiotics. After 3 weeks, she developed edema, sandpaper rash, lymphadenopathy, and hepatosplenomegaly. Lymph node biopsy revealed prominent paracortical hyperplasia and numerous viral inclusions in CD4positive $\mathrm{T}$ cells. Immunohistochemical and PCR studies confirmed infection with HHV-6. Patient was placed on steroids and the symptoms gradually resolved.

\section{References}

1 Yamanishi K, Okuno T, Shiraki K, et al. Identification of human herpesvirus- 6 as a causal agent for exanthem subitum. Lancet 1988;1:1065-1067.

2 Irving WL, Cunningham AL. Serological diagnosis of infection with human herpesvirus type 6. BMJ 1990; 300:156-159.

3 Merelli E, Sola P, Barozzi P, et al. An encephalitic episode in a multiple sclerosis patient with human herpesvirus 6 latent infection. J Neurol Sci 1996; 137:42-46.

4 Akashi K, Eizuru Y, Sumiyoshi Y, et al. Brief report: severe infectious mononucleosis-like syndrome and 
primary human herpesvirus 6 infection in an adult. N Engl J Med 1993;329:168-171.

5 Ablashi DV, Balachandran N, Josephs SF, et al. Genomic polymorphism, growth properties, and immunologic variations in human herpesvirus-6 isolates. Virology 1991;184:545-552.

6 Clark DA, Griffiths PD. Human herpesvirus 6: relevance of infection in the immunocompromised host. Br J Haematol 2003;120:384-395.

7 Di Luca D, Dolcetti R, Mirandola P, et al. Human herpesvirus 6: a survey of presence and variant distribution in normal peripheral lymphocytes and lymphoproliferative disorders. J Infect Dis 1994;170: 211-215.

8 Luppi M, Marasca R, Barozzi P, et al. Frequent detection of human herpesvirus-6 sequences by polymerase chain reaction in paraffin-embedded lymph nodes from patients with angioimmunoblastic lymphadenopathy and angioimmunoblastic lymphadenopathy-like lymphoma. Leuk Res 1993;17:1003-1011.

9 Levine $\mathrm{PH}$, Jahan N, Murari P, et al. Detection of human herpesvirus 6 in tissues involved by sinus histiocytosis with massive lymphadenopathy (RosaiDorfman disease). J Infect Dis 1992;166:291-295.

10 Luppi M, Barozzi P, Garber R, et al. Expression of human herpesvirus-6 antigens in benign and malignant lymphoproliferative diseases. Am J Pathol 1998; 153:815-823.

11 Lusso P, Malnati MS, Garzino-Demo A, et al. Infection of natural killer cells by human herpesvirus 6 . Nature 1993;362:458-462.

12 Lusso P, Markham PD, Tschachler E, et al. In vitro cellular tropism of human B-lymphotropic virus (human herpesvirus-6). J Exp Med 1988;167: 1659-1670.

13 Levy JA, Ferro F, Lennette ET, et al. Characterization of a new strain of HHV-6 (HHV-6SF) recovered from the saliva of an HIV-infected individual. Virology 1990; 178:113-121.

14 Kondo K, Kondo T, Okuno $\mathrm{T}$, et al. Latent human herpesvirus 6 infection of human monocytes/macrophages. J Gen Virol 1991;72(Pt 6): 1401-1408.

15 Ablashi DV, Lusso P, Hung CL, et al. Utilization of human hematopoietic cell lines for the propagation and characterization of HBLV (human herpesvirus 6). Int J Cancer 1988;42:787-791.
16 Quintanilla-Martinez L, Thieblemont C, Fend F, et al. Mantle cell lymphomas lack expression of p27Kip1, a cyclin-dependent kinase inhibitor. Am J Pathol 1998; 153:175-182.

17 McCarthy KP, Sloane JP, Kabarowski JH, et al. A simplified method of detection of clonal rearrangements of the T-cell receptor-gamma chain gene. Diagn Mol Pathol 1992;1:173-179.

18 Tang YW, Rys PN, Rutledge BJ, et al. Comparative evaluation of colorimetric microtiter plate systems for detection of herpes simplex virus in cerebrospinal fluid. J Clin Microbiol 1998;36:2714-2717.

19 Younes M, Podesta A, Helie M, et al. Infection of T but not B lymphocytes by cytomegalovirus in lymph node. An immunophenotypic study. Am J Surg Pathol 1991; 15:75-80.

20 Luppi M, Barozzi P, Morris C, et al. Human herpesvirus 6 latently infects early bone marrow progenitors in vivo. J Virol 1999;73:754-759.

21 Lusso P, Malnati M, De Maria A, et al. Productive infection of CD4+ and CD8+ mature human $\mathrm{T}$ cell populations and clones by human herpesvirus 6 Transcriptional down-regulation of CD3. J Immunol 1991;147:685-691.

22 Randhawa PS, Jenkins FJ, Nalesnik MA, et al. Herpesvirus 6 variant $A$ infection after heart transplantation with giant cell transformation in bile ductular and gastroduodenal epithelium. Am J Surg Pathol 1997;21:847-853.

23 Novoa LJ, Nagra RM, Nakawatase T, et al. Fulminant demyelinating encephalomyelitis associated with productive HHV-6 infection in an immunocompetent adult. J Med Virol 1997;52:301-308.

24 Roffman E, Albert JP, Goff JP, et al. Putative site for the acquisition of human herpesvirus 6 virion tegument. J Virol 1990;64:6308-6313.

25 Torrisi MR, Gentile M, Cardinali G, et al. Intracellular transport and maturation pathway of human herpesvirus 6. Virology 1999;257:460-471.

26 Granzow H, Weiland F, Jons A, et al. Ultrastructural analysis of the replication cycle of pseudorabies virus in cell culture: a reassessment. J Virol 1997;71: 2072-2082.

27 Hashimoto K, Yasukawa M, Tohyama M. Human herpesvirus 6 and drug allergy. Curr Opin Allergy Clin Immunol 2003;3:255-260. 